\title{
Clinical and Functional Outcomes of Implant Prostheses in Fibula Free Flaps
}

\author{
${ }^{1}$ Padma Ariga, ${ }^{2}$ Vinod Narayanan, ${ }^{3}$ Ashish R Jain, ${ }^{4}$ Jacob M Philip, ${ }^{5}$ Senthil Nathan
}

\section{ABSTRACT}

Introduction: Functional reconstruction of jaw defects due to surgical resection is a challenge in maxillofacial surgery. The fibula free flap in combination with dental implants has exhibited growing popularity for such reconstructions. This study aimed at evaluating the clinical and functional outcome of dental implants inserted in fibula free flaps and dental implants used for orofacial reconstruction following ablation of tumors.

Materials and methods: A clinical follow-up study of 10 patients was conducted after oral surgery, who received vascularized fibula bone grafts and endosseous implants for functional jaw reconstruction during a 12-year period. The follow-up protocol included a clinical examination and radiological evaluation and interview using a questionnaire. Information on postoperative recovery after the surgical reconstruction phase and implant placement, type of diet, swallowing problems, speech intelligibility, lip competence, and perceived facial appearance was collected and analyzed.

Results: The survival rate of fibula free flap was $100 \%$. The survival and success rates of implants placed in the fibula flaps at the end of the follow-up period were 100 and $94 \%$ respectively.

Conclusion: Implants placed in fibula bone grafts have been shown to integrate normally. Surgical reconstruction with vascularized fibula bone grafts followed by placement of dental implants after jaw resection can provide good clinical and functional outcomes for oral rehabilitation with a prosthesis.

Keywords: Bone grafts, Dental implants, Fibula free flap, Jaw reconstruction, Prosthesis.

How to cite this article: Ariga $P$, Narayanan V, Jain AR, Philip JM, Nathan S. Clinical and Functional Outcomes of Implant Prostheses in Fibula Free Flaps. World J Dent 2017;8(3):171-176.

\section{Source of support: Nil}

Conflict of interest: None

\footnotetext{
${ }^{1,3}$ Department of Prosthodontics, Saveetha Dental College and Hospitals, Saveetha University, Chennai, Tamil Nadu, India

${ }^{2,5}$ Department of Oral and Maxillofacial Surgery, Saveetha Dental College and Hospitals, Saveetha University, Chennai Tamil Nadu, India

${ }^{4}$ Department of Prosthodontics, Saveetha Dental College and Hospitals, Saveetha University, Chennai, Tamil Nadu, India Bharath University, Chennai, Tamil Nadu, India

Corresponding Author: Ashish R Jain, Department of Prosthodontics, Saveetha Dental College and Hospitals, Saveetha University, Chennai, Tamil Nadu, India, Phone: +919884233423 e-mail: dr.ashishjain_r@yahoo.com
}

\section{INTRODUCTION}

Surgical defects of the maxilla and mandible may give rise to various problems, such as disfigurement of facial contour, oroantral and oronasal fistulas, and impaired oral function. The use of revascularized free flaps has become a valuable means for rehabilitation of these patients.

Since its introduction by Taylor, ${ }^{1}$ the fibula free flap has become the most commonly used vascularized free flap used in the reconstruction of surgical defects, both of the mandible and the maxilla. ${ }^{2,3}$ It offers several desirable qualities for reconstruction, such as its length of 25 to $27 \mathrm{~cm}$, its vascular pedicle, and its ability to be bent to follow the shape of the interrupted mandible. It can be used as an osteomuscular flap or an osteomyocutaneous flap, providing the possibility of simultaneous reconstruction of deficient soft tissues on the intraoral side. The increasing popularity of its use has been reported in literature. ${ }^{4}$ Research in this field has mainly focused on the survival rate of the flap and postoperative complications. ${ }^{5-10}$ The ultimate goal of such a surgical reconstruction should be functional dental prosthetic rehabilitation, placing dental implants in the reconstructed area may overcome problems related to dental rehabilitation with removable prostheses. ${ }^{11-14}$ Fibular bone presents favorable conditions for implant placement, and subsequent implant supported prosthetic rehabilitation, due to its diameter and the good quality of its cortical bone. ${ }^{11,15-19}$ There are few studies focusing on the success rate of such treatment and the proportion of patients who are able to wear a functioning dental prosthesis after surgical reconstruction. Furthermore, patient perception about the functional outcome of such treatment has rarely been reported in studies. ${ }^{20-22}$ The purpose of this study was to evaluate the clinical and functional outcome of dental implants placed in a fibula free flap for jaw reconstruction. The objectives of this study were to evaluate:

- The survival rates of revascularized fibula flaps used for reconstruction of surgical defects of the maxilla and the mandible

- The survival and success rates of implants placed in these reconstructed areas

- The functional outcome of the prostheses retained/ supported by implants placed in these reconstructed areas, in terms of diet, mastication, speech, esthetics, and oral competence. 


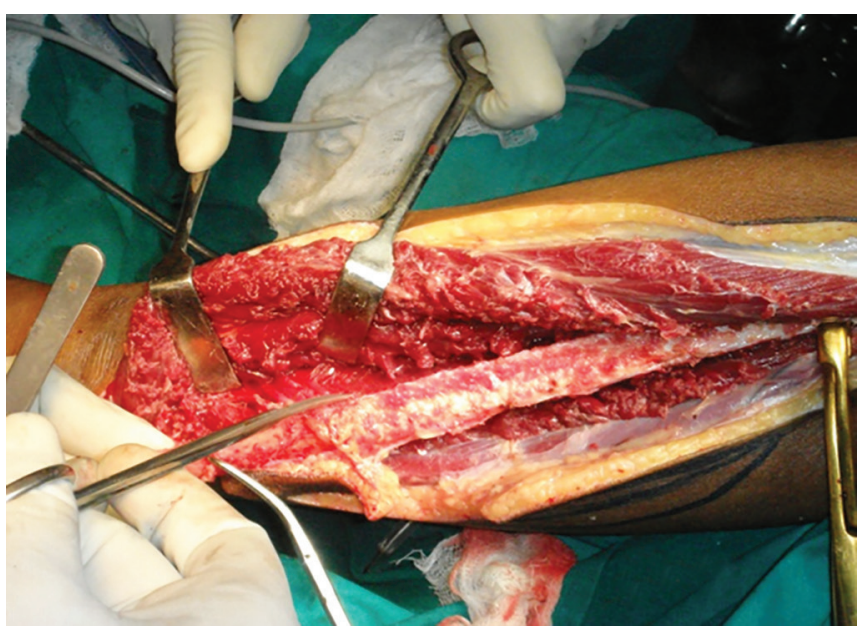

Fig. 1: Donor site - fibula free flap

\section{MATERIALS AND METHODS}

During a 12-year period (1998-2010), 10 patients $(n=10)$, 5 males and 5 females, aged between 18 and 60 years (mean age 29.9 years) underwent immediate reconstruction of surgical defects by means of revascularized fibula flaps (Figs 1 and 2, and Table 1).

Inclusion criteria for implant placement in the transplanted fibula were:

- A good prognosis after tumor resection

- Absence of recurrence signs

- Good oral hygiene

- Absence of periodontal disease in the residual dentition

- Request from the patient to be rehabilitated prosthetically.

Exclusion criteria for excluding patients from implant treatment were:

- Poor prognosis or systemically compromised health

- Patients with sufficient residual dentition to guarantee acceptable mastication

- Patients still abusing alcohol or smoking

- Noncompliant patients.

Among the 10 patients, 1 was fully edentulous and 9 were partially edentulous. A total of 33 implants were placed in 10 patients. A delayed loading protocol was

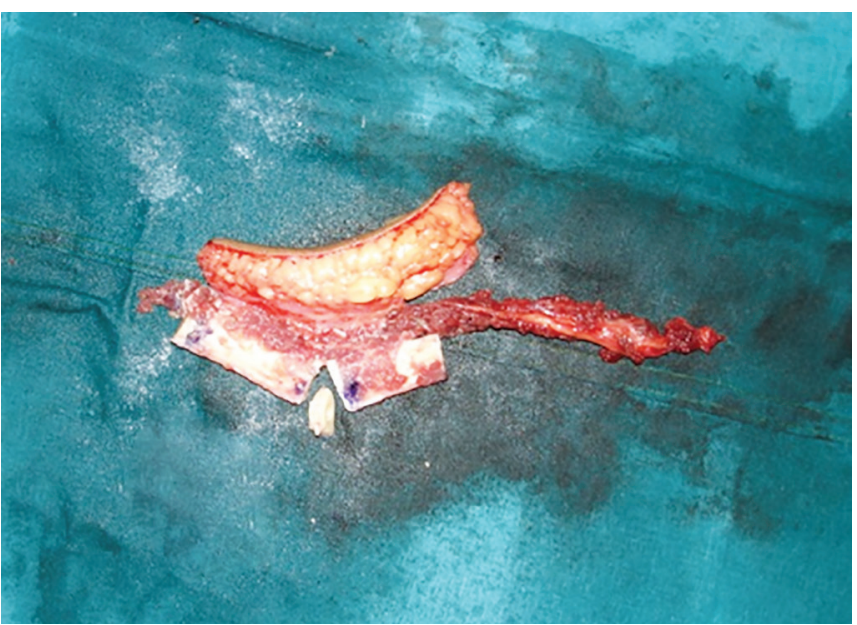

Fig. 2: Fibula free graft

followed (Table 2). The follow-up protocol was performed according to a standardized protocol including clinical examination, radiological examination, and interview using a questionnaire. The questionnaire covered the following aspects of reconstructive treatment: Postoperative recovery after the surgical reconstruction phase and implant placement, type of diet, swallowing problems, speech intelligibility, lip competence, and perceived facial appearance. The interview using this questionnaire was conducted in December 2010. Routine radiographic documentation of the patients was obtained with intraoral and panoramic radiographs taken preoperatively, immediately after implant placement, at the time of prosthetic rehabilitation, and annually thereafter (Figs 3 to 7). These were used to record periapical radiolucency and peri-implant bone resorption. Peri-implant bone resorption was recorded by comparing the periapical radiographs for bone-level changes mesial and distal to each implant, using a transparent millimeter ruler. The distance between the top of the implant head shoulder and the most coronal level of direct bone to implant contact was measured and compared with the baseline radiograph taken immediately after implant placement. The measurements were recorded to the nearest $0.5 \mathrm{~mm}$.

Table 1: Anagraphic data and clinical features of patients treated with fibula free flap

\begin{tabular}{clllll}
\hline Patient number & Age/Sex & Defect etiology & Site of reconstruction & Date of reconstruction & Residual dentition \\
\hline 1 & 45/M & Ameloblastoma & Mandible & November 1998 & Yes \\
2 & 19/F & Ameloblastoma & Mandible & February 2006 & Yes \\
3 & 19/F & Fibrous dysplasia & Maxilla & June 2006 & No \\
4 & 23/M & Ameloblastoma & Mandible & June 2007 & Yes \\
5 & 18/F & Ossifying fibroma & Maxilla & July 2007 & Yes \\
6 & 59/F & Osteoradionecrosis & Mandible & December 2007 & Yes \\
7 & 24/M & Ameloblastoma & Mandible & February 2008 & Yes \\
8 & 25/F & Odontogenic myxoma & Mandible & April 2008 & Yes \\
9 & 47/M & Central giant cell granuloma & Mandible & June 2008 & Yes \\
10 & 20/M & Central giant cell granuloma & Mandible & December 2008 & Yes \\
\hline
\end{tabular}


Clinical and Functional Outcomes of Implant Prostheses in Fibula Free Flaps

Table 2: Anagraphic data of patients treated with fibula free flap and dental implants

\begin{tabular}{rllll}
\hline Patient number & Number of implants & Date of implant placement & Date of restoration & Follow-up after restoration \\
\hline 1 & 4 & April 1999 & November 1999 & 140 \\
2 & 4 & March 2007 & June 2007 & 42 \\
3 & 4 & July 2007 & December 2008 & 18 \\
4 & 2 & July 2008 & January 2009 & 24 \\
5 & 2 & February 2009 & June 2009 & 18 \\
6 & 2 & March 2009 & October 2010 & 2 \\
7 & 6 & April 2009 & January 2010 & 12 \\
8 & 4 & November 2009 & July 2010 & 4 \\
9 & 2 & October 2009 & August 2010 & 3 \\
10 & 3 & May 2010 & October 2010 & 2 \\
\hline
\end{tabular}

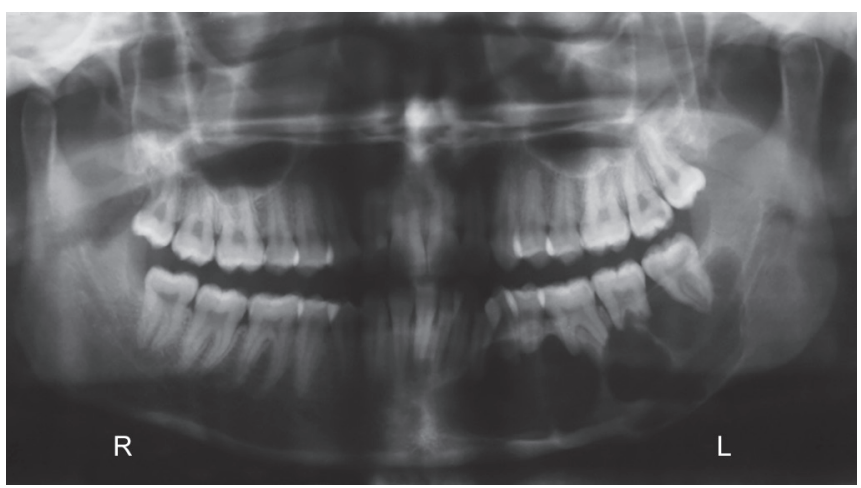

Fig. 3: Preoperative orthopantomogram

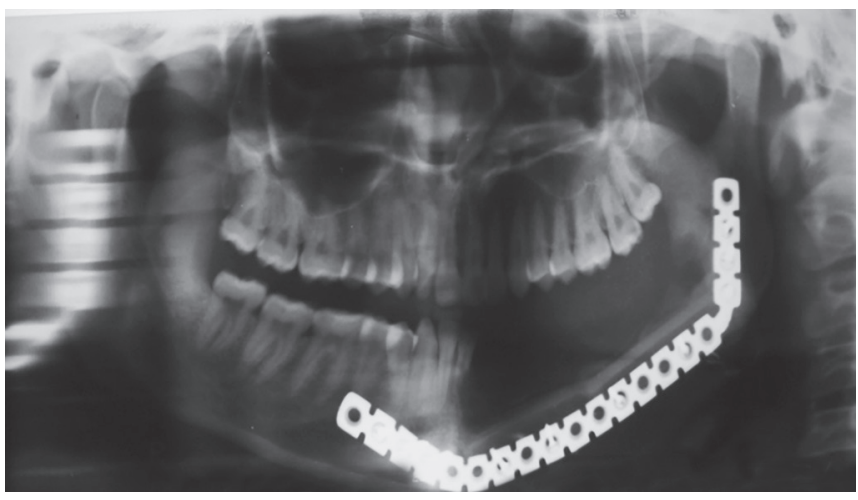

Fig. 5: Postoperative orthopantomogram after graft and plate placement

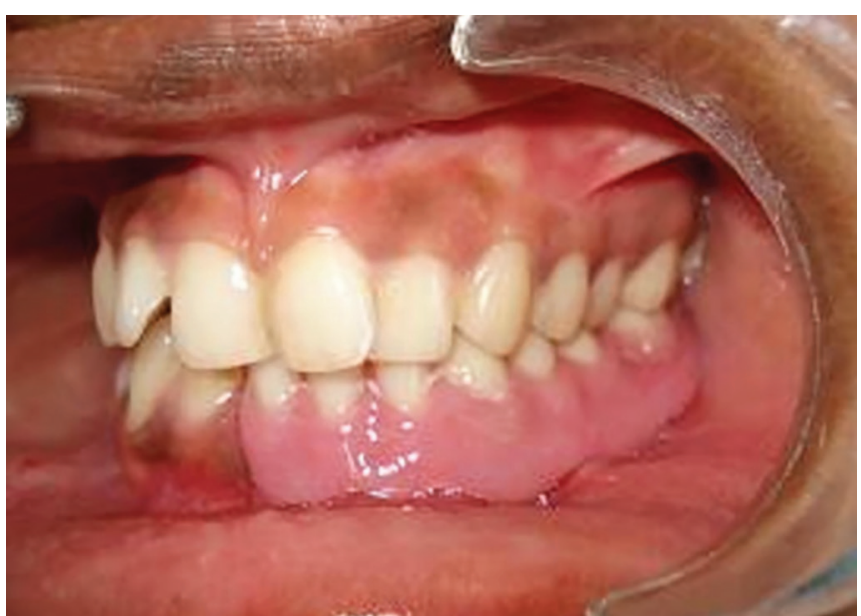

Fig. 7: Postinsertion of prosthesis

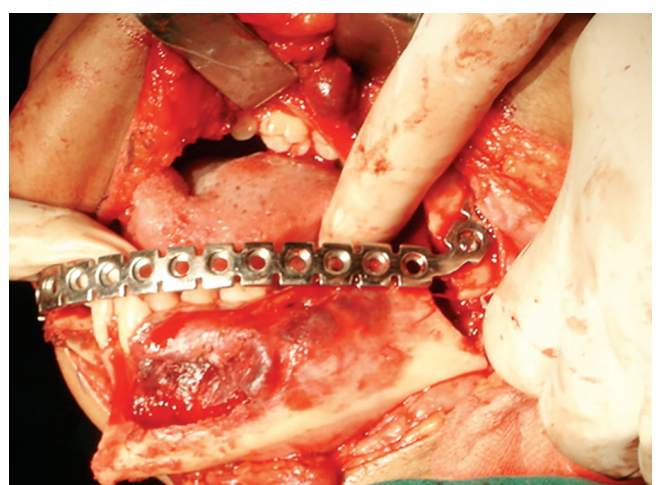

Fig. 4: Recipient site titanium plate and graft placement

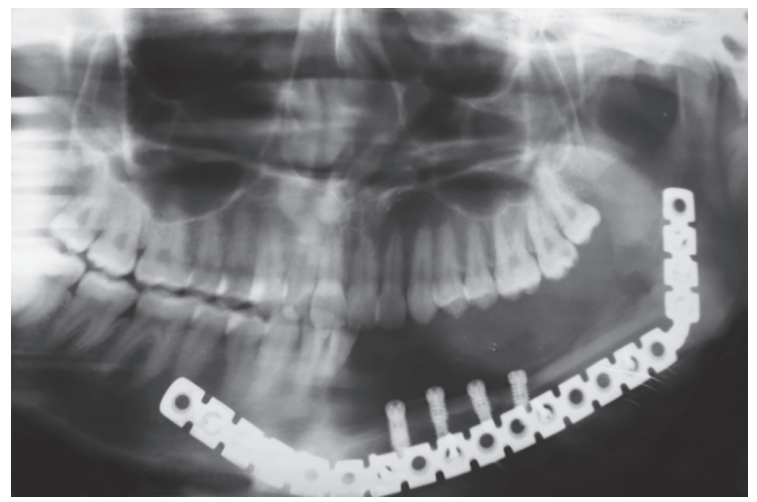

Fig. 6: Postoperative orthopantomogram after graft, plate placement with implant placement

A clinical examination was performed to record the presence or absence of (1) necrosis or nonintegration of the transplant with the recipient bone, (2) peri-implant infection with suppuration, and (3) implant mobility. The following classification was applied to group the functional results as categorical variables in the questionnaire: (1) Diet - normal diet, soft diet, or feeding tube, (2) speech - intelligible, intelligible with effort, or unintelligible, (3) oral competence - normal competence or drooling, (4) deglutition - normal or drooling, and (5) facial appearance - satisfactory or not satisfactory. The criteria for implant success include the following: (1) Absence of persistent pain, (2) absence of peri-implant infection with suppuration, (3) absence of mobility, 
(4) absence of continuous peri-implant radiolucency, and (5) peri-implant bone resorption $<1.5 \mathrm{~mm}$ in the first year of function and $<0.2 \mathrm{~mm}$ in subsequent years. The criteria for implant survival include the following: (1) Absence of persistent pain, (2) absence of peri-implant infection with suppuration, (3) absence of mobility, (4) absence of continuous peri-implant radiolucency, and (5) peri-implant bone resorption greater than values proposed by.

\section{RESULTS}

Primary reconstruction of surgical defects was performed for 10 patients with revascularized free flaps. A total of 33 implants were placed in these patients, 6 in the maxilla and 27 in the mandible. The mean time intervals between various stages of rehabilitation and follow-up are given in Table 3. Postoperative recovery after the surgical reconstruction phase was uneventful in 9 out of 10 patients, with excellent integration of the bone transplant and associated skin islands (when present) in the recipient bed. One flap underwent partial necrosis of the bone transplanted to the maxilla, but survived. Thus, the survival rate of fibula free flap was $100 \%$. Postoperative recovery after implant placement was uneventful in 9 out of 10 patients. The patient with the partially necrosed flap complained of persistent pain in both implants and presented peri-implant bone resorption values higher than those for implant success proposed. Thus, the survival and success rates of implants placed in the fibula flaps at the end of the follow-up period were 100 and $94 \%$ respectively. According to the functional evaluation, seven patients reported an improvement in dietary intake from a soft diet to a normal diet, after prosthetic rehabilitation. Seven patients reported normal masticatory ability while three reported difficulty in mastication. Nine of the patients were satisfied with the esthetic outcome after prosthodontic rehabilitation. Regarding speech, seven patients reported an improvement from being intelligible with effort to being intelligible after prosthodontic rehabilitation, while three of them reported no change, as their speech was intelligible before and after prosthodontic rehabilitation. Regarding oral competence, nine patients had normal continence and one suffered from drooling (Table 4). Patient distribution between diet and mastication showed that difficulty in mastication goes together with intolerance to normal diet. Only

Table 3: Mean time delay between treatment sequences

\begin{tabular}{ll}
\hline Time delay & Mean (months) \\
\hline Surgical reconstruction to implant placement & 13.4 \\
Implant placement to loading & 6.5 \\
Follow-up after loading & 36.5 \\
\hline
\end{tabular}

Table 4: Patient problems

\begin{tabular}{ll}
\hline Problem categories & $\begin{array}{l}\text { Number of } \\
\text { patients }(n=10)\end{array}$ \\
\hline Diet & $10 \%$ \\
Mastication & $80 \%$ \\
Speech & $0 \%$ \\
Facial appearance & $10 \%$ \\
Oral competence & $10 \%$ \\
No problems & $20 \%$ \\
Problems in four categories & $10 \%$ \\
\hline
\end{tabular}

one patient had the most severe disturbances of four of the evaluated oral functions including diet, mastication, facial appearance, and oral competence.

\section{DISCUSSION}

The fibula offers a reliable and satisfying reconstruction of maxillary and mandibular defects. The fibula has a biomechanical advantage in that it can favorably withstand masticatory loads. ${ }^{11,13,17,19,23,24}$ The morbidity in relation with the donor site was acceptable with limited pain and gait disturbances. Reconstruction of surgical jaw defects with fibula free flaps is not without problems. Debulking of the soft tissue component of the fibula placed for mandibular reconstruction was required for most patients to provide adequate interarch space to accommodate the prostheses. Seven out of 10 patients developed hyperplastic or inflammatory tissue response around the implant abutment causing pain and bleeding. This problem has been reported by other authors and may be a negative reaction of the skin around implants. ${ }^{25,26}$ It prevents proper seating of the prostheses and can be minimized by a well-contoured and -polished prostheses. Two methods of management of this problem has been reported in literature. ${ }^{27}$ This includes substitution of the skin around implants with mucosal grafts from the palate to obtain firmly attached, keratinized mucosa around implants, and the topical application of silver nitrate, particularly in patients who underwent maxillary resection, as this may lead to absence of palatal mucosa with no possibility of harvesting keratinized mucosa from this site. One limitation of the fibula free flap is that its average height is seldom more than $14 \mathrm{~mm}$, which leads to a step at the graft-to-residual stump interface, this can be avoided by fixation of the upper border of the flap at the level of the residual alveolar ridge. ${ }^{28}$ One problem with this technique is that it may negatively influence the profile of the lower border of the reconstructed mandible. Another solution is to use the double-barrel technique. ${ }^{29} \mathrm{~A}$ limitation of this technique is that the length of the bone segment may not be enough for the correction of large defects. The third solution may be a reconstructive procedure with a new revascularized flap fixed on top of the first one. A fourth 
possibility is by the use of vertical distraction osteogenesis to correct the height of the bone graft according to the level of the residual alveolar crest on the healthy side. Another limitation of the fibula is that when an osteocutaneous flap is used, it does not reestablish neuromuscular functions contributing to complex oral functions, which were lost during resection. The survival rate of fibula flaps in this case series was $100 \%$ which was higher than those results reported by other authors..$^{1-3,10,11,13,15,17,30} \mathrm{We}$ followed a delayed loading protocol for all implants in this case series although excellent primary stability has been cited as a reason for immediate loading of implants in fibula free flaps. ${ }^{31}$ Implant survival rate in our study was $100 \%$, which was higher than previous studies and implant success rate in our study was $94 \%$, which was lower than previous studies. ${ }^{10,19,23,24,26}$ Data regarding the usefulness of implant prosthodontic treatment in the functional rehabilitation of patients with jaw defects reconstructed with fibula free flaps are lacking. ${ }^{20}$ In this study, $80 \%$ of the patients could not chew properly on both sides although only one patient had limitations in the consistency of food she could consume. In a study by Leung and Cheung, ${ }^{32} 32.1 \%$ of dentally reconstructed patients had no limitations on food consistency and $28.6 \%$ complained about problems associated with instability of their dental prosthesis. In this study, $20 \%$ of the patients had no functional problems with their dental prosthesis. This was consistent with the results obtained in one other study, but was higher than those of other reports which ranged between 0 and $12 \%{ }^{7,9,10,20}$ There is no general agreement that endosseous implants should fail to a higher degree due to irradiation. ${ }^{33}$ Radiation exceeding 50 Gy can cause destruction of capillaries, proliferative endarteritis, damage to osteoprogenitor cells, and overall reduced neovascularity, which can impair the process of osseointegration. ${ }^{34}$ Even if implants are placed before radiation therapy, irradiated tissues which contain endosseous implants increase the risk of soft tissue dehiscences around the implants, and infection which may lead to loss of the implants. ${ }^{35}$ In a study by Iizuka et al, ${ }^{20} 10$ patients with 13 implants received postoperative radiotherapy, there was no problems related to implant stability or implant loss on follow-up. This supports the view that implant installation is not contraindicated for cancer patients who undergo subsequent radiation therapy. In this study, one patient underwent radiotherapy before implant placement and did not present with problems related to implant stability or implant loss on follow-up. Although the number of cases we report was not sufficient to allow any definite conclusion, our results at least support the view that implant installation is not contraindicated for cancer patients who underwent previous radiation therapy.

\section{CONCLUSION}

The fibula free flap presents many advantages compared with the scapula or the iliac crest. It has a high survival rate. The use of vascularized bone grafts have contributed to the high rate of implant success and survival. Implant-supported dental prosthetic rehabilitation positively influences oral function. The results of the present study indicate that implants placed in vascularized fibula grafts following jaw resection is a viable treatment option for oral rehabilitation. Despite some persistent soft tissue problems and implant loss, most patients demonstrated successful clinical and functional prosthetic outcomes.

\section{REFERENCES}

1. Taylor GI. Reconstruction of the mandible with free composite iliac bone grafts. Ann Plast Surg 1982 Nov;9(5):361-376.

2. Wolff KD, Ervens J, Herzog K, Hoffmeister B. Experience with the osteocutaneous fibula flap: an analysis of 24 consecutive reconstructions of composite mandibular defects. J Craniomaxillofac Surg 1996 Dec;24(6):330-338.

3. Ferri J, Caprioli F, Peuvrel G, Langlois JM. Use of the fibula free flap in maxillary reconstruction: a report of 3 cases. J Oral Maxillofac Surg 2002 May;60(5):567-574.

4. Jones NF, Swartz WM, Mears DC, Jupiter JB, Grossman A. The "double barrel" free vascularized fibular bone graft. Plast Reconstr Surg 1988 Mar;81(3):378-385.

5. Riediger D. Restoration of masticatory function by microsurgically revascularized iliac crest bone grafts using enosseous implants. Plast Reconstruct Surg 1988 Jun;81(6):861-877.

6. Jewer DD, Boyd JB, Manktelow RT, Zuker RM, Rosen IB, Gullane PJ, Rotstein LE, Freeman JE. Orofacial and mandibular reconstruction with the iliac crest free flap: a review of 60 cases and a new method of classification. Plast Reconstr Surg 1989 Sep;84(3):391-403.

7. Komisar A. The functional result of mandibular reconstruction. Laryngoscope 1990 Apr;100(4):364-374.

8. Urken ML, Weinberg H, Vickery C, Buchbinder D, Lawson W, Biller HF. Oromandibular reconstruction using microvascular composite free flaps. Report of 71 cases and a new classification scheme for bony, soft-tissue, and neurologic defects. Arch Otolaryngol Head Neck Surg 1991 Jul;117(7):733-744.

9. Shpitzer T, Neligan PC, Gullane PJ, Freeman JE, Boyd BJ, Rotstein LE, Brown DH, Irish JC, Gur E. Oromandibular reconstruction with the fibular free flap. Analysis of 50 consecutive flaps. Arch Otolaryngol Head Neck Surg 1997 Sep;123(9):939-944.

10. Gürlek A, Miller MJ, Jacob RF, Lively JA, Schusterman MA. Functional results of dental restoration with osseointegrated implants after mandible reconstruction. Plast Reconstruct Surg 1998 Mar;101(3):650-655.

11. Moscoso JF, Keller J, Genden E, Weinberg H, Biller HF, Buchbinder D, Urken ML. Vascularized bone flaps in oromandibular reconstruction. A comparative anatomic study of bone stock from various donor sites to assess suitability for enosseous dental implants. Arch Otolaryngol Head Neck Surg 1994 Jan;120(1):36-43.

12. Buchbinder D, Urken ML, Vickery C, Weinberg H, Sheiner A, Biller H. Functional mandibular reconstruction of patients 
with oral cancer. Oral Surg Oral Med Oral Pathol 1989 Oct;68(4 Pt 2):499-503.

13. SchmelzeisenR,NeukamFW,Shirota T,SpechtB,WichmannM. Postoperative function after implant insertion in vascularized bone grafts in maxilla and mandible. Plast Reconstr Surg 1996 Apr;97(4):719-725.

14. Pogrel MA, Podlesh S, Anthony JP, Alexander J. A comparison of vascularized and nonvascularized bone grafts for reconstruction of mandibular continuity defects. J Oral Maxillofac Surg 1997 Nov;55(11):1200-1206.

15. Flemming AF, Brough MD, Evans ND, Grant HR, Harris M, James DR, Lawlor M, Laws IM. Mandibular reconstruction using vascularized fibula. Br J Plast Surg 1990 Jul;43(4): 403-409.

16. Barber HD, Seckinger RJ, Hayden RE, Weinstein GS. Evaluation of osseointegration of endosseous implants in radiated, vascularized fibula flaps to the mandible: a pilot study. J Oral Maxillofac Surg 1995 Jun;53(6):640-644.

17. Hayter JP, Cawood JI. Oral rehabilitation with endosteal implants and free flaps. Int J Oral Maxillofac Surg 1996 Feb;25(1):3-12.

18. Chiapasco, M. Implants for patients with maxillofacial defects and following irradiation. In: Lang, NL.; Karring, T.; Lindhe, J., editors. Proceedings III European Workshop on Periodontology. Berlin: Quintessence; 1999. p. 557-607.

19. Chiapasco M, AbatiS, Ramundo G, Rossi A, Romeo E, Vogel G. Behavior of implants in bone grafts or free flaps after tumor resection. Clin Oral Implants Res 2000 Feb;11(1):66-75.

20. Iizuka T,Häfliger J,Seto I, Rahal A, Mericske-Stern R,Smolka K. Oral rehabilitation after mandibular reconstruction using an osteocutaneous fibula free flap with endosseous implants. Factors affecting the functional outcome in patients with oral cancer. Clin Oral Implants Res 2005 Feb;16(1):69-79.

21. Matsui Y, Neukam FW, Schmelzeisen R, Ohno K. Masticatory function of postoperative tumor patients rehabilitated with osseointegrated implants. Int J Oral Maxillofac Surg 1996 Apr;54(4):441-447.

22. Sloan JA, Tolman DE, Anderson JD, Sugar AW, Wolfaardt JF, Novotny P. Patients with reconstruction of craniofacial or intraoral defects: development of instruments to measure quality of life. Int J Oral Maxillofac Implants 2001 Mar-Apr;16(2): 225-245.

23. Chan MF, Hayter JP, Cawood JI, Howell RA. Oral rehabilitation with implant-retained prostheses following ablative surgery and reconstruction with free flaps. Int J Oral Maxillofac Implants 1997 Nov-Dec;12(6):820-827.
24. Roumanas ED, Markowitz BL, Lorant JA, Calcaterra TC, Jones NF, Beumer J 3rd. Reconstructed mandibular defects: fibula free flaps and osseointegrated implants. Plast Reconstr Surg 1997 Feb;99(2):356-365.

25. Rohner D, Kunz C, Bucher P, Hammer B, Prein J. New possibilities for reconstructing extensive jaw defects with prefabricated microvascular fibula transplants and ITI implants. Mund Kiefer Gesichtschir 2000 Nov;4(6):365-372.

26. Jaquiéry C, Rohner D, Kunz C, Bucher P, Peters F, Schenk RK, Hammer, B. Reconstruction of maxillary and mandibular defects using prefabricated microvascular fibular grafts and osseointegrated dental implants - a prospective study. Clin Oral Implants Res 2004 Oct;15(5):598-606.

27. Chiapasco M, Biglioli F, Autelitano L, Romeo E, Brusati R. Clinical outcome of dental implants placed in fibula-free flaps used for the reconstruction of maxillo-mandibular defects following ablation for tumors or osteoradionecrosis. Clin Oral Implants Res 2006 Apr;17(2):220-228.

28. Chang YM, Santamaria E, Wei FC, Chen HC, Chan CP, Shen YF, Hou SP. Primary insertion of osseointegrated dental implants into fibula osteoseptocutaneous free flap for mandible reconstruction. Plast Reconstr Surg 1998 Sep;102(3): 680-688.

29. Bähr W, Stoll P, Wächter R. Use of the "double barrel" free vascularized fibula in mandibular reconstruction. J Oral Maxillofac Surg 1998 Jan;56(1):38-44.

30. Bianchi B, Ferrari S, Poli T, Bertolini F, Raho T, Sesenna E. Oromandibular reconstruction with simultaneous free flaps: experience on 10 cases. Acta Otorhinolaryngol Ital 2003 Aug;23(4):281-290.

31. Chiapasco M, Gatti C. Immediate loading of dental implants placed in revascularized fibula free flaps: a clinical report on 2 consecutive patients. Int J Oral Maxillofac Implants 2004 Nov-Dec;19(6):906-912.

32. Leung AC, Cheung LK. Dental implants in reconstructed jaws: patients' evaluation of functional and quality-of-life outcomes. Int J Oral Maxillofac Implants 2003 Jan-Feb;18(1):127-134.

33. Granström G. Radiotherapy, osseointegration and hyperbaric oxygen therapy. Periodontol 2000 2003;33:145-162.

34. Granström G, Bergström K, Tjellström A, Brånemark PI. A detailed analysis of titanium implants lost in irradiated tissues. Int J Oral Maxillofac Implants 1994;9:653-662.

35. Granström G, Tjellström A, Brånemark PI, Fornander J. Bone-anchored reconstruction of the irradiated head and neck cancer patient. Otolaryngol Head Neck Surg 1993 Apr;108(4):334-343. 